\title{
Indirect insulin resistance detection: Current clinical trends and laboratory limitations
}

\author{
Sylwia Placzkowska a , Lilla Pawlik-Sobecka ${ }^{\mathrm{b}}$, Izabela Kokot ${ }^{\mathrm{b}}$, Agnieszka Piwowarc
}

There is a steady increase in the number of overweight and obese people worldwide and increasingly, younger people. Excess adipose tissue impairs the action of insulin, leading to insulin resistance (IR). Tissue IR is a major factor in relation to cardiovascular disease, metabolic syndrome and diabetes. Thus, it is important to recognize at the pre-disease stage with the possibility of therapeutic intervention. IR is assessed using indicators of epidemiological significance, most often calculated from fasting and postprandial glucose and insulin values, so-called indirect indicators of insulin resistance. The most commonly used parameter is the Homeostatic Model Assessment (HOMA). Although the Quantitative Insulin Sensitivity Check Index (QUICKI), Matsuda Index and the Insulin Secretion-Sensitivity Index-2 (ISSI-2) are also used, the values of these indices established for IR vary for different age, sex, populations and ethnic groups. Thus, appropriate reference values of indirect indices should be determined for such groups, and when this is precluded, data from published studies carried out on the most ethnically, socio-economically and age-matched populations should be applied.

Key words: insulin resistance, reference interval, decision limit

Received: November 8, 2018; Revised: April 25, 2019; Accepted: May 7, 2019; Available online: June 4, 2019 https://doi.org/10.5507/bp.2019.021

(c) 2019 The Authors. This is an open access article licensed under the Creative Commons Attribution License (https://creativecommons.org/licenses/by/4.0/).

\begin{abstract}
${ }^{a}$ Diagnostics Laboratory for Teaching and Research, Faculty of Pharmacy with the Division of Laboratory Diagnostics, Wroclaw Medical University, Wroclaw, Poland

${ }^{b}$ Department of Laboratory Diagnostics, Faculty of Pharmacy with the Division of Laboratory Diagnostics, Wroclaw Medical University, Wroclaw, Poland

'Department of Toxicology, Faculty of Pharmacy with the Division of Laboratory Diagnostics, Wroclaw Medical University, Wroclaw, Poland Corresponding author: Izabela Kokot, e-mail: izabela.kokot@umed.wroc.pl
\end{abstract}

\section{BACKGROUND}

Insulin resistance (IR) is a pathological state of disturbance between insulin synthesis and its action in the tissues. In this condition, tissue sensitivity to insulin is reduced, which results in impaired glucose homeostasis ${ }^{1}$. Insulin resistance causes a broad spectrum of clinical symptoms, and participates in many pathological states, such as obesity, glucose intolerance, diabetes mellitus (DM), metabolic syndrome (MS), and cardiovascular diseases (CVD). Many of these disorders are associated with various endocrine, metabolic, and genetic factors, but the influences of individual, socio-economic and dietary factors are also indicated ${ }^{2}$. Current research shows that insulin resistance affects a large middle-aged population of working-age and is particularly associated with overweight and obesity ${ }^{3}$. It is estimated that in highly developed countries as many as $15.5-51.0 \%$ of adults are affected by IR $\left(\right.$ ref. $\left.^{46}\right)$. However, recent studies have shown that different metabolic disturbances and insulin resistance may also affect young, apparently healthy people with normal body weight and no overt metabolic disorders, as was confirmed in our own research ${ }^{7}$. Different indirect indices are applied for the purpose of insulin resistance recognition, among them those calculated from fasting glucose and insulin concentration, as well as those derived from its measurement during the oral glucose tolerance test (OGTT) (ref. ${ }^{8,9}$ ). There are few published studies on decision limits for insulin resistance indices, so this condition is recognized with different frequencies even based on the same indices. The most important factors influencing indices values are age, ethnicity origin and lifestyle, as well as the laboratory methods used for glucose and insulin concentration determination ${ }^{10}$. Because of the lack of official guidelines, efforts towards the determination of reference intervals of insulin resistance indices are still necessary for proper diagnosis ${ }^{11}$. For this reason, it is crucially necessary to define reference values for the indices used in IR diagnosis. This may enable the early detection of metabolic disorders and the introduction of preventive actions, especially for young people.

Establishing reference intervals or decision limits could facilitate the use of indirect insulin resistance indices in routine clinical practice, due to accurate identification of individuals at risk for metabolic diseases and the introduction of personalised therapeutic interventions ${ }^{12,13}$. It is especially important for the practices of primary care physicians and family doctors, with regard to the increased prevalence of insulin resistance in the world population ${ }^{14}$. The aim of this review is to provide information about the most popularly used indirect indices of insulin resistance, insulin sensitivity and beta cell capac- 
ity, such as HOMA1-IR, HOMA2, HOMA2 C-peptide, QUICKI, MATSUDA and ISSI-2, and their values used in the recognition of IR, in the context of their clinical usefulness as well as their laboratory limitations.

\section{INSULIN RESISTANCE RECOGNITION}

The problem of IR occurrence and its appropriate and early detection is especially important given the increasingly commonly observed trend of metabolic disorders in developed countries. The "gold standard" of IR recognition is represented by the glucose clamp test or the frequently-sampled intravenous glucose tolerance test (FSIVGTT), with glucose and/or insulin infusion. Due to their highly invasive and time-consuming nature, the use of these tests in clinical practice is significantly limited ${ }^{15}$.

Measurements of glucose and insulin in the fasting state and during OGTT are commonly used to diagnose carbohydrate disturbances and detect insulin resistance. The most common method of IR diagnosis is based on the measurement of blood glucose and insulin concentrations ${ }^{16}$. Detected abnormalities in these parameters may be the first sign of impaired metabolic homeostasis and an indication for further detailed diagnosis, i.e. the performance of additional tests and the calculation of indirect insulin resistance indices. Indirect indices of insulin resistance have been used to assess not only cell resistance to insulin, but also the function and insulin sensitivity of pancreatic beta cells. The most widely used indirect indices are derived from Homeostatic Model Assessment (HOMA), Quantitative Insulin Sensitivity Check Index (QUICKI), Matsuda Index ${ }^{8}$ and Insulin SecretionSensitivity Index-2 (ISSI-2) ( ref. $^{17}$ ). Current criteria for diagnosing carbohydrate metabolism disorders are well known and are published annually by national diabetes associations, such as the American Diabetes Association ${ }^{18}$, the Japanese Diabetes Association ${ }^{19}$ and Diabetes Poland $^{20}$. However there are still no clear guidelines for everyday clinical diagnosis of the insulin resistance which precedes carbohydrate metabolism development.

\section{CURRENTLY USED INDIRECT INSULIN RESISTANCE INDICES}

\section{Insulin/Glucose Index (I/GI)}

Because of its simplicity in carrying out and calculation, one of the most popular indices used in clinical practice for IR recognition is the insulin/glucose index (I/GI), calculated on the basis of insulin $[\mathrm{mU} / \mathrm{L}]$ and glucose $[\mathrm{mg} / \mathrm{dL}]$ concentrations in the fasting state. The index cannot, however, be applied on patients with abnormalities of endogenous insulin secretion ${ }^{21}$.

\section{Homeostatic Model Assessment (HOMA)}

The Homeostatic Model Assessment (HOMA) is a mathematical model originally developed by David Matthews et al. in 1985. HOMA is used to quantify insulin resistance (HOMA1-IR), sensitivity (HOMA-\%S) and $\beta$-cell function (HOMA-\%B). It is calculated on the basis of fasting glucose and insulin or C-peptide concentrations. The relationship of fasting glucose to fasting insulin reflects the balance between hepatic glucose production and insulin secretion, maintained by the feedback loop between the liver and pancreatic beta cells ${ }^{8,12}$. The primary HOMA model expresses the glucose-insulin balance in a simple nonlinear mathematical equation that does not take into account peripheral or hepatic insulin resistance and correlates well with the glycemic clamp ${ }^{22}$. It is calculated on the basis of the following formulas ${ }^{23}$ :

HOMA1-IR $=\frac{\text { fasting blood glucose }\left[\frac{\mathrm{mmol}}{\mathrm{L}}\right] * \text { fasting insulin }\left[\frac{\mathrm{mU}}{\mathrm{L}}\right]}{22,5}$

When the traditional units for glucose $(\mathrm{mg} / \mathrm{dL})$ are used, the denominator value of the formula is 405 .

When HOMA-\%S is used to estimate the sensitivity of tissues to insulin, which is the inverse of the HOMA1-IR equation:

HOMA-\%S $=\frac{1}{\text { HOMA1-IR }}$

For the estimation of pancreatic beta cell function the HOMA-\%B index is used, calculated from the following equation:

$$
\text { HOMA-\%B }=\frac{20 * \text { fasting insulin }\left[\frac{\mathrm{mU}}{\mathrm{L}}\right]}{\text { fasting blood glucose }\left[\frac{\mathrm{mmol}}{\mathrm{L}}\right]-3,5}
$$

In 1996, Levy et al. updated the HOMA model to one known as HOMA2, which takes into account hepatic and peripheral insulin resistance. Additionally, this modification of the original HOMA equation includes renal glucose losses, enabling more accurate use in hyperglycaemic patients. This version also incorporates proinsulin secretion into the model. Therefore it enables the use of both total insulin determined with radioimmunoassay (RIA) and insulin, excluding proinsulin, measured with the enzyme-linked immunosorbent assay method (ELISA). HOMA2 is a fully computerised model, based on a nonlinear equation that has not yet been published. Levy's model can be used to determine insulin sensitivity $(\% \mathrm{~S})$ and beta-cell function $(\% \mathrm{~B})$ from fasting blood glucose and insulin, as well as C-peptide concentrations (HOMA C-pept.) (ref. ${ }^{24}$ ). The use of insulin concentrations for HOMA index calculations may be biased, due to partial hepatic insulin metabolism, so C-peptide levels seem to better reflect the actual index value, since its stability in blood is longer ${ }^{12}$. The HOMA2 model can be used across a range of 20-400 pmol/L (2.9-57.6 IU/mL) for insulin and 54.1-450.5 mg/dL (3.5-25.0 $\mathrm{mmol} / \mathrm{L})$ for glucose concentration in patients' blood ${ }^{25}$.

\section{Quantitative Insulin Sensitivity Check Index (QUICKI)}

QUICKI was first published in 2000 by Katz et al., and used for insulin sensitivity estimation. It is an empirically derived mathematical transformation of fasting glucose and fasting insulin concentrations, and correlates well with the glycemic clamp. This index is particularly applicable for obese and diabetic patients. However, its 
reliability is limited in subjects with type 1 diabetes mellitus. Quantitative Insulin Sensitivity Check Index is a variation of the HOMA equation, and for its calculation the following formula is used (ref. ${ }^{26,27}$ ):

QUICKI $=\frac{1}{\log \text { fasting insulin }\left[\frac{\mu \mathrm{U}}{\mathrm{mL}}\right]+\log \text { fasting glucose }\left[\frac{\mathrm{mg}}{\mathrm{dL}}\right]}$

\section{Matsuda Index}

The Matsuda index was developed and published in 1999 by Matsuda et al. to assess total, whole-body insulin sensitivity. This index is calculated from plasma glucose

100000

Matsuda Index $\sqrt{\text { fasting insulin }\left[\frac{\mu \mathrm{U}}{\mathrm{mL}}\right] * \text { fasting glucose }\left[\frac{\mathrm{mg}}{\mathrm{dL}}\right] * \text { area under the insulin OGTT curve }\left[\frac{\mu \mathrm{U}}{\mathrm{mL}}\right] * \text { area under glucose OGTT curve }\left[\frac{\mathrm{mg}}{\mathrm{dL}}\right]}$

and insulin concentrations obtained from fasting blood samples, and after oral ingestion of $75 \mathrm{~g}$ glucose during the OGTT. The index is derived from the following formula ${ }^{28}$ :

\section{Insulin Secretion-Sensitivity Index-2 (ISSI-2)}

The Disposition Index was described by Steven E. $\mathrm{Kahn}^{29}$ in 1993. It evaluates pancreatic beta-cell function after intravenous glucose load and refers to the relationship between insulin sensitivity and insulin secretion, which is a constant value for people with the same level of glucose tolerance. Primarily this index was calculated from insulin sensitivity and insulin secretion values, derived from Frequently Sampled Intravenous Glucose Tolerance Test results. This test, developed by Bergman et al. ${ }^{30}$, involves the intravenous injection of glucose and multiple glucose and insulin blood sampling, thus evaluating peripheral and hepatic insulin sensitivity. The Disposition Index can also be calculated from values obtained by the euglycemic clamp. In this model, a peripheral insulin sensitivity value is obtained, therefore, in order to quantify the Disposition Index, hepatic insulin sensitivity should also be estimated.

In many epidemiological and clinical studies, the values obtained from OGTT are used to determine the Disposition Index. The use of indices of beta cells compensation calculated from OGTT results require a hyperbolic relationship between insulin sensitivity and secretion. Therefore, in recent years new methods of evaluating the beta-cell functions were developed, which are analogous to the Disposition Index ${ }^{31-33}$. One of them, published by Retnakaran et al. ${ }^{34}$ in 2009 , is the Insulin Secretion-Sensitivity Index-2 (ISSI-2). The ISSI-2 is quantified from the area-under-the-insulin-curve (AUCins) and the area-under-the-glucose-curve (AUCgluc) during the extended to minimum 3 blood examination oral glucose tolerance test. The obtained AUCins and AUCgluc ratio is then multiplied by the above mentioned Matsuda index. The Insulin Secretion-Sensitivity Index-2 is calculated from the formula:

$$
\text { ISSI-2 }=\frac{\mathrm{AUC}_{\text {ins } 0-120}}{\mathrm{AUC}_{\text {gluc } 0-120}} \quad * \quad \text { Mat }
$$

However, the final result of ISSI- 2 calculations is dependent on the applied glucose and insulin units. To compare values between studies, this should be taken into consideration. Moreover, different methods may be used for AUC calculation ${ }^{35}$, so the ISSI-2 values obtained from the same results can differ due to the calculation methods applied.

\section{Choosing a proper index}

We presented in this review only those insulin resistance and beta cell efficiency indirect indices which have been well described in the literature and used in clinical practice.

Such a variety of IR indices puts the researcher and the clinician before the choice of the most appropriate IR indices for clinical purpose, when the most reliable metabolic clamp cannot be performed or is not recommended for the patient ${ }^{28,36}$. Borai et al ${ }^{37}$ showed that the methods based on the above-described glucose and insulin measurements both under fasting and glucose loading are the most useful and ultimately the only one to use in routine practice.

However, due to the unavoidable biological and analytical variability of glucose, and in particular insulin determinations ${ }^{38}$, indicators based on a repeated glucose and insulin measurements obtained during the OGTT test give a more precise estimation of the severity of insulin resistance and beta cell efficiency than those calculated only in fasting state ${ }^{39}$.

Although, in general, during diabetes development, early onset of peripheral insulin resistance before hepatic insulin resistance is indicated ${ }^{40}$, our observations suggest that insulin resistance may be reflected in varying degrees in elevated fasting and post-load insulin and glucose concentrations. In our pilot study among young people, we recognized both the liver and peripheral insulin resistance with different frequency ${ }^{41}$. However, euglycemic fasting and impaired glucose tolerance patients were observed at the same time and vice versa. Therefore, in our opinion, the calculation of at least two or more indicators for the same patient also increases the clinical usefulness of the information obtained due to the possibility to deter- 
mine the dominant type of insulin resistance, its main mechanism and tissues affected (central, e.g. HOMA 2, QUICKI, peripheral e.g. Matsuda, beta cell performance e.g. ISSI-2) (ref. $\left.{ }^{42}\right)$. However, it should be emphasized that in the development of diabetes, patients progress from hyperinsulinemia to the level of exhaustion of pancreatic beta cells secretory reserves. In these patients, despite insulin resistance, slightly higher or even normal insulin concentrations may be observed, especially in the postprandial state ${ }^{33}$. Therefore, in such causes the indexes calculated in the fasting state will be much more reliable than those calculated after loading, which is a condition requiring the activation of pancreatic secretory reserves.

In monitoring the development of insulin resistance and the effectiveness of therapeutic interventions in order to reduce it, it is important to calculate indicators determined with at least the same analytical methods, and preferably laboratory systems (method and analyzer). This is due to the still insufficient standardization of insulin determinations and the sensitivity of immunochemical methods to internal and external confounders, and con- sequently to significant interlaboratory differences in the determinations of this parameter.

The mathematical model used to calculate the indicators is also important for the final result. Subsequent modifications and up-grade mathematical formulas, most often based on the use of additional variables or logarithmic' transformation of values. Those mathematical operations allow us to consider factors such as hyperglycemia, hyperinsulinaemia and non-Gaussian distribution of results in the general population in final calculation. The aim of such modifications is to increase the correlation of the obtained results with the gold standard (which is a metabolic clamp) and if such a goal is achieved, it is recommended to use modified indicators such as HOMA2 and QUICKI (ref. ${ }^{12}$ ).

To the best of our knowledge, physicians in clinical practice and a large number of dietitians, most often use the HOMA index in its original version described by Matthews et al. ${ }^{23}$. This is also confirmed by the fact that HOMA is the most frequently used indicator in epidemiological studies. In the literature, the cut off for IR

Table 1. Procedural steps of reference intervals determination according to CLSI recommendations.

\begin{tabular}{|c|c|}
\hline Procedural Steps & Terms and Definitions \\
\hline $\begin{array}{l}\text { 1. Establish a list of potential analytical interferences based on the medical } \\
\text { and scientific literature. } \\
\text { 2. Establish acceptance or exclusion criteria for the potential reference indi- } \\
\text { vidual as well as an appropriate questionnaire. } \\
\text { 3. Execute written consent and completed questionnaire from the research } \\
\text { participant. } \\
\text { 4. Categorize the potential reference individuals on the basis of the personal } \\
\text { information questionnaire findings and the results of their health assessment. }\end{array}$ & $\begin{array}{l}\text { Reference population consists of reference } \\
\text { individuals: people selected for comparison } \\
\text { with the use of defined criteria }\end{array}$ \\
\hline $\begin{array}{l}\text { 5. Exclude individuals from the sample group based on the exclusion criteria } \\
\text { or results of assessment indicating ill health. } \\
\text { 6. Establish an appropriate number of reference individuals, taking into account } \\
\text { the desired confidence limits. } \\
\text { 7. Properly and consistently prepare each individual for specimen collection for } \\
\text { the measurements performed in the accordance with the routine practice. } \\
\text { 8. Collect the biological specimens properly and handle them in a manner } \\
\text { consistent with the routine procedure for patient specimens. } \\
\text { 9. Collect the reference values by analyses of the specimens in accordance with } \\
\text { the appropriate methodology, under well-defined measurement conditions } \\
\text { and consistent with the routine practice for patient specimens. }\end{array}$ & $\begin{array}{l}\text { Reference sample group: } \\
\text { a number of reference individuals statistically } \\
\text { adequate to represent the reference popula- } \\
\text { tion }\end{array}$ \\
\hline $\begin{array}{l}\text { 10. Inspect the obtained reference values and prepare a histogram in order to } \\
\text { evaluate the data distribution. } \\
\text { 11. Identify potential outliers and data errors. }\end{array}$ & $\begin{array}{l}\text { Reference values: } \\
\text { value obtained from a reference sample sub- } \\
\text { ject by adequate observation or measurement }\end{array}$ \\
\hline $\begin{array}{l}\text { 12. Analyse the reference values, select an estimation method and estimate refer- } \\
\text { ence limits as well as the reference intervals. If necessary, include a partition } \\
\text { of subjects into subgroups for separate reference ranges. }\end{array}$ & $\begin{array}{l}\text { Reference distribution: } \\
\text { statistical distribution of reference values }\end{array}$ \\
\hline 13. Document the particular stages and all the performed procedures. & $\begin{array}{l}\text { Reference limit: } \\
\text { a limit used for descriptive purposes, defined } \\
\text { on the basis of reference distribution } \\
\text { Reference interval: } \\
\text { the range of values within the reference lim- } \\
\text { its, which usually includes } 95 \% \text { of observed } \\
\text { values }\end{array}$ \\
\hline
\end{tabular}


Table 2. The values of insulin resistance indices designated for different populations using different classification criteria.

\begin{tabular}{|c|c|c|c|c|c|}
\hline Author (year) Ref. & $\begin{array}{l}\text { 1. Study population } \\
\text { 2. Subject number }(\mathrm{n}) \\
\text { 3. Sex proportion }(\mathrm{W} / \mathrm{M}) \\
\text { 4. Age (years) mean } \pm \mathrm{SD} \\
\text { (interval) }\end{array}$ & $\begin{array}{l}\text { Method determination of insulin (manufacturer) } \\
\text { Method determination of glucose (manufacturer) }\end{array}$ & $\begin{array}{l}\text { Method of decision values } \\
\text { determination }\end{array}$ & $\begin{array}{l}\text { Estimated insulin } \\
\text { resistance indices }\end{array}$ & Designated value \\
\hline $\begin{array}{l}\text { 1. Graffigna et al., } 53 \\
\text { (2005) }\end{array}$ & $\begin{array}{l}\text { 1. Argentinean-Spanish origin } \\
\text { young adults } \\
\text { 2. } \mathrm{n}=363 \\
\text { 3. } 95 \mathrm{~W} \text { and } 268 \mathrm{M} \\
\text { 4. } 37 \pm 12 \mathrm{~W}, 36 \pm 11 \mathrm{M} \text { years } \\
(18-65)\end{array}$ & $\begin{array}{l}\text { Insulin - chemiluminescent method - CLIA } \\
\text { (Immulite) } \\
\text { Glucose - enzymatic colorimetric method } \\
\text { (no data provided) }\end{array}$ & Mean \pm SD & HOMA1-IR & $\begin{array}{l}2.04 \pm 1.77 \text { for all } \\
1.87 \pm 1.72 \text { for } \mathrm{W} \\
2.09 \pm 1.79 \text { for } \mathrm{M}\end{array}$ \\
\hline $\begin{array}{l}\text { 2. Szurkowska et } \\
\text { al., (2005) }\end{array}$ & $\begin{array}{l}\text { 1. Polish adults with NGT and } \\
\text { BMI }<25 \mathrm{~kg} / \mathrm{m}^{2} \\
\text { 2. } \mathrm{n}=2838 \text { all population } \\
\text { (a reference group - no data } \\
\text { provided) } \\
\text { 3. No data provided } \\
\text { 4. - }(35-75) \text { years }\end{array}$ & $\begin{array}{l}\text { Insulin - immunoradiometric method (IRMA, } \\
\text { Swierk) }\end{array}$ & $\begin{array}{l}\text { Cut-off for insulin resistance } \\
\text { identification on: } \\
75^{\text {th }} \text { percentile } \\
25^{\text {th }} \text { percentile }\end{array}$ & $\begin{array}{l}\text { HOMA1-IR } \\
\text { QUICKI } \\
\text { Matsuda index }\end{array}$ & $\begin{array}{l}>2.1 \\
<0.34 \\
<7.3\end{array}$ \\
\hline $\begin{array}{l}\text { 3. Tresaco et al., } \\
\text { (2005) }\end{array}$ & $\begin{array}{l}\text { 1. Spanish children with and } \\
\text { without obesity } \\
\text { 2. } \mathrm{n}=140 \text { ( } 72 \text { non-obese, } 68 \text { with } \\
\text { exogenous obesity) } \\
\text { 3. } 68 \mathrm{~W} \text { and } 72 \mathrm{M} \\
\text { 4. } 11.01 \pm 2.13 \text { years }(7-16)\end{array}$ & $\begin{array}{l}\text { Glucose - enzymatic colorimetric assay (Roche/ } \\
\text { Hitachi) }\end{array}$ & $\begin{array}{l}\text { ROC curve for } \\
\text { MS identification } \\
\text { Cut-off for insulin resistance } \\
\text { identification on: } \\
75^{\text {th }} \text { percentile }\end{array}$ & 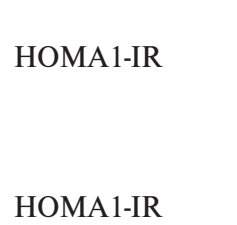 & 2.28 \\
\hline $\begin{array}{l}\text { 4. Keskin et al., } \\
\text { (2005) }\end{array}$ & $\begin{array}{l}\text { 1. Turkish children and adolescents } \\
\text { with pubertal obesity } \\
\text { 2. } \mathrm{n}=57 \\
\text { 3. } 30 \mathrm{~W} \text { and } 27 \mathrm{M} \\
\text { 4. } 12.04 \pm 2.90 \text { years }\end{array}$ & $\begin{array}{l}\text { Insulin - radioimmunoassay method (no data } \\
\text { provided) } \\
\text { Glucose - oxidase method (no data provided) }\end{array}$ & $\begin{array}{l}\text { ROC curve for insulin } \\
\text { resistance identification }\end{array}$ & HOMA1-IR & 3.16 \\
\hline $\begin{array}{l}\text { 5. Geloneze et al., } 57 \\
\text { (2009) }\end{array}$ & $\begin{array}{l}\text { 1. Nondiabetic Brazilians } \\
\text { 2. } \mathrm{n}=1203 \\
\text { 3. } 692 \mathrm{~W} \text { and } 511 \mathrm{M} \\
\text { 4. median } 41 \\
\text { (Q1-Q3: } 32-50) \text { years (18-78) }\end{array}$ & $\begin{array}{l}\text { Insulin - radioimmunoassay method (Linco } \\
\text { Research Inc.) } \\
\text { Glucose - oxidase method (no data provided) }\end{array}$ & $\begin{array}{l}\text { ROC curve for MS } \\
\text { identification } \\
\text { Cut-off for insulin resistance } \\
\text { identification on: } \\
90^{\text {th }} \text { percentile } \\
\text { ( } \mathrm{n}=297 \text {; selected healthy } \\
\text { group) }\end{array}$ & $\begin{array}{l}\text { HOMA1-IR } \\
\text { HOMA2 }\end{array}$ & $\begin{array}{l}2.7 \\
1.8\end{array}$ \\
\hline
\end{tabular}


Table 2. Continued.

\begin{tabular}{|c|c|c|c|c|c|c|}
\hline \multirow[t]{2}{*}{$\begin{array}{l}\text { 6. d'Annunzio et } \\
\text { al., (2009) }\end{array}$} & 58 & $\begin{array}{l}\text { 1. Italian healthy children and } \\
\text { adolescents } \\
\text { 2. } \mathrm{n}=142 \\
\text { 3. } 57 \mathrm{~W} \text { and } 85 \mathrm{M} \\
\text { 4. } 10.6 \pm 3.8 \text { years }(2.7-19)\end{array}$ & $\begin{array}{l}\text { Insulin - radioimmunoassay method } \\
\text { (Radim Kit, Rome) }\end{array}$ & Mean \pm SD & $\begin{array}{l}\text { HOMA1-IR } \\
\text { QUICKI }\end{array}$ & $\begin{array}{l}1.49 \pm 0.91 \text { for all } \\
1.37 \pm 0.73 \text { for } \mathrm{M} \\
1.65 \pm 1.10 \text { for } \mathrm{W} \\
\\
0.37 \pm 0.04 \text { for all } \\
0.38 \pm 0.04 \text { for } \mathrm{M} \\
0.37 \pm 0.04 \text { for } \mathrm{W}\end{array}$ \\
\hline & & & $\begin{array}{l}\text { Glucose - oxidase method } \\
\text { (no data provided) }\end{array}$ & & НОМА- $\beta \%$ & $\begin{array}{l}144.1 \pm 142.2 \text { for all } \\
130.4 \pm 124.7 \text { for } \mathrm{M} \\
164.6 \pm 163.9 \text { for } \mathrm{W}\end{array}$ \\
\hline $\begin{array}{l}\text { 7. Gayoso-Diz } \\
\text { et al., } \\
\text { (2011) }\end{array}$ & 59 & $\begin{array}{l}\text { 1. Non-diabetic Spanish subjects } \\
\text { 2. } \mathrm{n}=2246 \\
\text { 3. } 1329 \mathrm{~W} \text { and } 917 \mathrm{M} \\
\text { 4. Median } 47 \text { years }(20-92)\end{array}$ & $\begin{array}{l}\text { Insulin - radioimmunoassay method } \\
\text { (no data provided) } \\
\text { Glucose - enzymatic hexokinase method } \\
\text { (no data provided) }\end{array}$ & Median & HOMA1-IR & $\begin{array}{l}1.73 \text { for all } \\
2.06 \text { for } \mathrm{M} \\
1.93 \text { for } \mathrm{W}\end{array}$ \\
\hline \multirow[t]{2}{*}{$\begin{array}{l}\text { 8. Yamada et al., } \\
\text { (2011) }\end{array}$} & 51 & $\begin{array}{l}\text { 1. Selected Japanese reference } \\
\text { individuals (with } \mathrm{NFG} \text {, } \\
\left.\text { BMI }<25 \mathrm{~kg} / \mathrm{m}^{2}, \text { ALT }<31 \mathrm{U} / \mathrm{L}\right) \\
\text { 2. } \mathrm{n}=2153 \\
\text { 3. } 1336 \mathrm{~W} \text { and } 817 \mathrm{M} \\
\text { 4. } 46.0 \pm 11.0 \text { year }(20-79 \text { ) years }\end{array}$ & $\begin{array}{l}\text { Insulin - fluorescence-enzyme method } \\
\text { (ST AIA-PACK IRI; Toso, Tokyo) }\end{array}$ & $\begin{array}{l}\text { Reference interval according } \\
\text { to CLSI (C28-A3) between } \\
2.5^{\text {th }}-97.5^{\text {th }} \text { percentile } \\
\text { (RI; mean } \pm 2 \text { SD of log } \\
\text { HOMA1-IR values) }\end{array}$ & HOMA1-IR & $0.4-2.4$ \\
\hline & & & Glucose - (no data provided) & $\begin{array}{l}\text { Cut-off for insulin resistance } \\
\text { identification }\end{array}$ & HOMA1-IR & $\geq 2.5$ \\
\hline $\begin{array}{l}\text { 9. Qu et al., } \\
\text { (2011) }\end{array}$ & 6 & $\begin{array}{l}\text { 1. Mexican Americans } \\
\text { 2. } n=1854 \\
\text { 3. No data provided } \\
\text { 4. } \geq 18 \text { years }\end{array}$ & $\begin{array}{l}\text { Insulin - enzyme-linked immunosorbent assay/ } \\
\text { ELISA immunoassay kit (Mercodia, Uppsala) } \\
\text { Glucose - method - no data provided, (Glucostat } \\
\text { analyzer (Model 27, YSI, IncYellow Springs, Ohio) }\end{array}$ & $\begin{array}{l}\text { ROC curve for insulin } \\
\text { resistance identification }\end{array}$ & HOMA1-IR & 3.80 \\
\hline $\begin{array}{l}\text { 10. Oka et al., } \\
\text { (2012) }\end{array}$ & 60 & $\begin{array}{l}\text { 1. Middle-aged Japanese with NGT } \\
\text { 2. } \mathrm{n}=1125 \\
\text { 3. } 697 \mathrm{M} \text { and } 428 \mathrm{~W} \\
\text { 4. } 51.9 \pm 7.6 \text { years }(30-65)\end{array}$ & $\begin{array}{l}\text { Insulin -chemiluminescence immunoassay method } \\
\text { (BML, Inc. Tokyo) } \\
\text { Glucose - oxidase method (Automatic Glucose } \\
\text { Analyzer ADAMS Glucose GA-1160, Arkray, } \\
\text { Kyoto) }\end{array}$ & Mean $(95 \%$ CI $)$ & $\begin{array}{l}\text { HOMA1-IR } \\
\text { Matsuda ISI } \\
\text { Disposition Index } \\
\text { (ISSI-2)* }\end{array}$ & $\begin{array}{l}0.83(0.81-0.86) \\
14.8(14.1-15.4) \\
2.92(2.79-3.03)\end{array}$ \\
\hline \multirow[t]{2}{*}{$\begin{array}{l}\text { 11. Würtz et al., } \\
\text { (2012) }\end{array}$} & 61 & $\begin{array}{l}\text { 1. Young adults Finns } \\
\text { 2. } \mathrm{n}=7098 \\
\text { 3. } 3665 \mathrm{~W} \text { and } 3433 \mathrm{M} \\
\text { 4. } 31 \pm 3 \text { years }(24-39)\end{array}$ & $\begin{array}{l}\text { Insulin - radioimmunoassay } \\
\text { (Pharmacia Diagnostics)/ microparticle enzyme } \\
\text { immunoassay kit (Abbott Laboratories) }\end{array}$ & Median (Q1-Q3) & HOMA1-IR & $\begin{array}{l}0.98(0.78-1.30) \text { for } \mathrm{M} \\
0.92(0.73-1.20) \text { for } \mathrm{W}\end{array}$ \\
\hline & & & $\begin{array}{l}\text { Glucose - glucose dehydrogenase } \\
\text { (Granustest 250; Diagnostica Merck)/ } \\
\text { enzymatically (Olympus AU400) }\end{array}$ & $\begin{array}{l}\text { Cut-off for insulin resistance } \\
\text { identification on: } \\
80^{\text {th }} \text { percentile }\end{array}$ & HOMA1-IR & 1.3 \\
\hline
\end{tabular}


Table 2. Continued.

\begin{tabular}{|c|c|c|c|c|c|c|}
\hline $\begin{array}{l}\text { 12. Yamada et al., } \\
\text { (2012) }\end{array}$ & & $\begin{array}{l}\text { 1. Non-diabetic Japanese } \\
\text { 2. } \mathrm{n}=6868 \\
\text { 3. } 3141 \mathrm{~W} \text { and } 3727 \mathrm{M} \\
\text { 4. } 49.3 \pm 11.7 \text { years for } \mathrm{W} \\
\text { and } 49.7 \pm 12.1 \text { years for } \mathrm{M}\end{array}$ & $\begin{array}{l}\text { Insulin - fluorescence-enzyme method (ST AIA- } \\
\text { PACK IRI; Toso, Tokyo) } \\
\text { Glucose - no data provided }\end{array}$ & $\begin{array}{l}\text { ROC curve for MS } \\
\text { identification }\end{array}$ & HOMA1-IR & 1.70 \\
\hline $\begin{array}{l}\text { 13. Stankiewicz- } \\
\text { Olczyk et al., } \\
(2012)\end{array}$ & 63 & $\begin{array}{l}\text { 1. Polish professionally active men } \\
\text { 2. } \mathrm{n}=402 \\
\text { 3. } 402 \mathrm{M} \\
\text { 4. } 30-60 \text { years }\end{array}$ & Insulin - no data provided & Mean \pm SD & HOMA1-IR & $\begin{array}{l}2.49 \pm 1.93 \\
\text { Men without MS } \\
1.74 \pm 1.16 \\
\text { Men with MS } \\
3.41 \pm 2.38\end{array}$ \\
\hline & & & Glucose - no data provided & & QUICKI & $\begin{array}{l}0.34 \pm 0.03 \\
\text { Men without MS } \\
0.36 \pm 0.03 \\
\text { Men with MS } \\
0.32 \pm 0.02 \\
\end{array}$ \\
\hline $\begin{array}{l}\text { 14. Takahara et al., } \\
\text { (2013) }\end{array}$ & 52 & $\begin{array}{l}\text { 1. Healthy reference Japanese } \\
\text { subjects } \\
\text { 2. } \mathrm{n}=204 \\
\text { 3. } 60 \mathrm{~W} \text { and } 144 \mathrm{M} \\
\text { 4. } 49 \pm 9 \text { years }(23-69)\end{array}$ & $\begin{array}{l}\text { Insulin - no data provided } \\
\text { Glucose - no data provided }\end{array}$ & $\begin{array}{l}\text { One-sided reference interval } \\
\text { according to CLSI as: } \\
\text { Mean +2SD } \\
\text { Mean -2SD } \\
\text { Insulin resistance } \\
\text { identification according to } \\
\text { CLSI, as: } \\
\text { Mean -2SD } \\
\text { Mean +2SD }\end{array}$ & $\begin{array}{l}\text { Matsuda Index } \\
\text { HOMA1-IR }\end{array}$ & $\begin{array}{l}\geq 4.3 \\
\leq 2.4\end{array}$ \\
\hline $\begin{array}{l}\text { 15. Kozakowski et } \\
\text { al., (2013) }\end{array}$ & 64 & $\begin{array}{l}\text { 1. Polish PCOS women } \\
\text { 2. } \mathrm{n}=40 \\
\text { 3. } 40 \mathrm{~W} \\
\text { 4. } 28.6 \pm 7.6 \text { years }(19-49)\end{array}$ & $\begin{array}{l}\text { Insulin - immunoradiometric method } \\
\text { (Immunotech SA, France) } \\
\text { Glucose - hexokinase method (Cobas Integra 400, } \\
\text { Roche Diagnostics) }\end{array}$ & Mean \pm SD & HOMA1-IR & $2.68 \pm 2.4$ \\
\hline $\begin{array}{l}\text { 16. Gayoso-Diz et } \\
\text { al., (2013) }\end{array}$ & 65 & $\begin{array}{l}\text { 1. Spanish general adult population } \\
\text { 2. } \mathrm{n}=2459 \\
\text { 3. } 1436 \mathrm{~W} \text { and } 1023 \mathrm{M} \\
\text { 4. } 49.4 \pm 16.2 \text { years }(20-92)\end{array}$ & $\begin{array}{l}\text { Insulin - radioimmunoassay method } \\
\text { (Coat A Count Insulin, Los Angeles) } \\
\text { Glucose - hexokinase enzymatic method } \\
\text { (no data provided) }\end{array}$ & $\begin{array}{l}\text { Cut-off for insulin resistance } \\
\text { identification on: } \\
90^{\text {th }} \text { percentile }\end{array}$ & HOMA1-IR & 3.46 \\
\hline $\begin{array}{l}\text { 17. Timoteo et al., } \\
\text { (2014) }\end{array}$ & 66 & $\begin{array}{l}\text { 1. Portuguese patients admitted } \\
\text { in a Cardiology ward } \\
\text { 2. } \mathrm{n}=1784 \\
\text { 3. } 874 \mathrm{~W} \text { and } 910 \mathrm{M} \\
\text { 4. } 58.1 \pm 18.2 \text { year (no data) }\end{array}$ & $\begin{array}{l}\text { Insulin - electrochemiluminescence method - } \\
\text { ECLIA (no data provided) } \\
\text { Glucose - the glucose-oxidase method } \\
\text { (no data provided) }\end{array}$ & $\begin{array}{l}\text { Cut-off for insulin resistance } \\
\text { identification on: } \\
90^{\text {th }} \text { percentile } \\
\text { ROC curve for MS } \\
\text { identification }\end{array}$ & HOMA1-IR & $2.41($ for $n=300)$ \\
\hline
\end{tabular}


Table 2. Continued.

\begin{tabular}{|c|c|c|c|c|c|c|}
\hline $\begin{array}{l}\text { 18. Tohidi et al., } \\
\text { (2014) }\end{array}$ & 9 & $\begin{array}{l}\text { 1. Non-obese healthy Iranian with } \\
\mathrm{FSI}<2.88 \mu \mathrm{U} / \mathrm{mL} \text {, } \\
\mathrm{FG}<3.5 \mathrm{mmol} / \mathrm{L} \\
\text { 2. } \mathrm{n}=275 \\
\text { 3. } 167 \mathrm{~W} \text { and } 108 \mathrm{M} \\
\text { 4. } 34.5 \pm 7.5 \text { years for } \mathrm{W} \text { and } \\
41.3 \pm 13.9 \text { years for } \mathrm{M}(24-83)\end{array}$ & $\begin{array}{l}\text { Insulin - electrochemiluminescence immunoassay } \\
\text { - ECLIA (Roche Diagnostics kit/ Cobas e-411 } \\
\text { analyzer, Roche/Hitachi, GmbH, Mannheim) } \\
\text { Glucose - oxidase method (no data provided) }\end{array}$ & $\begin{array}{l}\text { Reference interval according } \\
\text { to CLSI/IFCC between: } \\
2.5^{\text {th }}-97.5^{\text {th }} \text { percentile }\end{array}$ & $\begin{array}{l}\text { HOMA1-IR } \\
\text { HOMA2 } \\
\text { QUICKI }\end{array}$ & $\begin{array}{l}0.63-2.68 \\
0.40-1.80 \\
0.33-0.42\end{array}$ \\
\hline $\begin{array}{l}\text { 19. Skoczeń et al., } \\
\text { (2014) }\end{array}$ & 67 & $\begin{array}{l}\text { 1. Polish children with simple } \\
\text { obesity } \\
\text { 2. } \mathrm{n}=222 \\
\text { 3. } 109 \mathrm{~W} \text { and } 113 \mathrm{M} \\
\text { 4. } 13.1 \pm 3.7 \text { years }(2-18) \\
\end{array}$ & $\begin{array}{l}\text { Insulin - no data provided } \\
\text { Glucose - no data provided }\end{array}$ & Mean \pm SD & $\begin{array}{l}\text { HOMA1-IR } \\
<10 \text { years, } \\
10-16 \text { years, } \\
>16 \text { years }\end{array}$ & $\begin{array}{l}1.6 \pm 1.2 \\
3.6 \pm 4.6 \\
2.3 \pm 1.7\end{array}$ \\
\hline $\begin{array}{l}\text { 20. Bednarek- } \\
\text { Tupikowska et } \\
\text { al., (2014) }\end{array}$ & 68 & $\begin{array}{l}\text { 1. Polish adults with } \mathrm{BMI}<27 \mathrm{~kg} / \mathrm{m}^{2} \\
\text { 2. } \mathrm{n}=342 \\
\text { 3. } 218 \mathrm{~W} \text { and } 124 \mathrm{M} \\
\text { 4. } 20-40 \text { years }\end{array}$ & $\begin{array}{l}\text { Insulin - Micro Particle Enzyme Immunoassay } \\
\text { (AxSym Insulin Kit, Abbott) } \\
\text { Glucose - oxidase method } \\
\text { (Dade Behring Marburg GmbH) }\end{array}$ & Mean \pm SD & HOMA1-IR & $\begin{array}{l}\text { Non-obese normal } \\
\text { subjects } \\
1.07 \pm 0.43 \\
\text { MONW subjects } \\
2.33 \pm 0.77\end{array}$ \\
\hline & & & & & QUICKI & $\begin{array}{l}\text { Non-obese normal } \\
\text { subjects } \\
0.40 \pm 0.05 \\
\text { MONW subjects } \\
0.31 \pm 0.03\end{array}$ \\
\hline & & & & & FIRI & $\begin{array}{l}\text { Non-obese normal } \\
\text { subjects } \\
0.95 \pm 0.35 \\
\text { MONW subjects } \\
1.49 \pm 0.87\end{array}$ \\
\hline $\begin{array}{l}\text { 21. Oh et al., } \\
\text { (2015) }\end{array}$ & 69 & $\begin{array}{l}\text { 1. Korean adults with NGT and } \\
\text { glucose concentration } \\
<155 \mathrm{mg} / \mathrm{dl} \text { in } 60 \text { minute OGTT } \\
\text { 2. } \mathrm{n}=149 \\
\text { 3. } 117 \mathrm{~W} \text { and } 32 \mathrm{M} \\
\text { 4. } 52.8 \pm 7.0 \text { years }(30-80)\end{array}$ & $\begin{array}{l}\text { Insulin - no data provided } \\
\text { Glucose - no data provided }\end{array}$ & Mean \pm SD & $\begin{array}{l}\text { Disposition Index (ISSI-2) } \\
\text { HOMA1-IR } \\
\text { HOMA- } \beta \text {-cell } \\
\text { Matsuda Index }\end{array}$ & $\begin{array}{l}301.2 \pm 113.7 \\
1.2 \pm 0.6 \\
102.7 \pm 52.9 \\
9.3 \pm 4.9\end{array}$ \\
\hline $\begin{array}{l}\text { 22. González- } \\
\text { Zavala et al., } \\
\text { (2015) }\end{array}$ & 70 & $\begin{array}{l}\text { 1. Mexican adolescents in different } \\
\text { pubertal stages } \\
\text { 2. } \mathrm{n}=292 \\
\text { 3. } 152 \mathrm{~W} \text { and } 140 \mathrm{M} \\
\text { 4. } 13.02 \pm 0.94 \text { years }(12-15)\end{array}$ & $\begin{array}{l}\text { Insulin - immunofluorescence assay with labeled } \\
\text { substrate - IFALS (TOSOH AIA-600, Tokyo) } \\
\text { Glucose - hexokinase enzymatic method } \\
\text { (InCCA - Intelligent Clinical Chemistry Analyzer, } \\
\text { Diconex) }\end{array}$ & Mean \pm SD & HOMA1-IR & $\begin{array}{l}2.9 \pm 2.5 \text { - } \text { all subjects } \\
2.3 \pm 1.3 \text { - Prepubertal } \\
2.8 \pm 2.4 \text { - } \text { Middle } \\
\quad \text { pubertal } \\
3.3 \pm 3.0 \text { - Postpubertal }\end{array}$ \\
\hline
\end{tabular}


Table 2. Continued.

\begin{tabular}{|c|c|c|c|c|c|c|}
\hline $\begin{array}{l}\text { 23. Santos et al., } \\
\text { (2016) }\end{array}$ & & $\begin{array}{l}\text { 1. Chilean NGT adults with FPG } \\
<100 \mathrm{mg} / \mathrm{dL} \text { and } 2 \text {-h glucose } \\
\text { OGTT levels }<140 \mathrm{mg} / \mathrm{dL} \\
\text { 2. } \mathrm{n}=1393 \\
\text { 3. } 1178 \mathrm{~W} \text { and } 215 \mathrm{M} \\
\text { 4. } 36.5 \pm 11.1 \text { years }(18-60)\end{array}$ & $\begin{array}{l}\text { Insulin -electrochemiluminescence immunoassay } \\
\text { (no data provided) } \\
\text { Glucose - colorimetric } \\
\text { glucose-oxidase method (no data provided) }\end{array}$ & Mean \pm SD & $\begin{array}{l}\text { ISSI-2 * } \\
\text { Matsuda ISI-COMP index } \\
\text { HOMA-S index }\end{array}$ & $\begin{array}{l}2.73 \pm 1.10 \\
4.7 \pm 2.8 \\
63.8 \pm 39.1\end{array}$ \\
\hline $\begin{array}{l}\text { 24. Kwon et al., } \\
\text { (2017) }\end{array}$ & (1) & $\begin{array}{l}\text { 1. Young healthy Korean } \\
\text { 2. } \mathrm{n}=8707 \\
\text { 3. } 4515 \mathrm{~W} \text { and } 4192 \mathrm{M} \\
\text { 4. } 45.63 \pm 0.23 \mathrm{M}, 44.31 \pm 0.21 \mathrm{~W} \\
\text { years, }(20-39)\end{array}$ & $\begin{array}{l}\text { Insulin - radioimmunoassy (Gamma counter; } \\
\text { Hewlett Packard, USA/ } 1470 \text { WIZARD gamma- } \\
\text { counter (PerkinElmer) } \\
\text { Glucose - method - no data provided; ADVIA } \\
\text { 1650, (Siemens, USA)/ Hitachi } 7600 \text { (Hitachi) }\end{array}$ & $\begin{array}{l}\text { Cut-off for insulin resistance } \\
\text { identification on: } \\
75^{\text {th }} \text { percentile }\end{array}$ & HOMA1-IR & $\begin{array}{l}2.18 \text { for } \mathrm{W} \\
2.19 \text { for } \mathrm{M}\end{array}$ \\
\hline $\begin{array}{l}\text { 25. Płaczkowska } \\
\text { et al., (2019) }\end{array}$ & 72 & $\begin{array}{l}\text { 1. Polish young adults } \\
\text { 2. } \mathrm{n}=130 \\
\text { 3. } 106 \mathrm{~W} \text { and } 24 \mathrm{M} \\
\text { 4. Median age } 23 \text { years } \\
\text { (Q1-Q3: } 21-24)(18-31)\end{array}$ & $\begin{array}{l}\text { Insulin - enzyme-linked immunosorbent assay } \\
\text { (ELISA, DRG Diagnostics) } \\
\text { Glucose - GOD/POD method } \\
\text { (Thermo Electron Oy, Vantaa) }\end{array}$ & $\begin{array}{l}\text { One-sided reference interval } \\
\text { according to CLSI as: } \\
95^{\text {th }} \text { percentile } \\
5^{\text {th }} \text { percentile } \\
\text { Cut-off for insulin resistance } \\
\text { identification on: } \\
75^{\text {th }} \text { percentile } \\
25^{\text {th }} \text { percentile }\end{array}$ & $\begin{array}{l}\text { HOMA1-IR } \\
\text { HOMA2 } \\
\text { QUICKI } \\
\text { Matsuda } \\
\text { ISSI-2 }\end{array}$ & $\begin{array}{l}\leq 4.00 \\
\leq 2.27 \\
\geq 0.31 \\
\geq 3.19 \\
\geq 206\end{array}$ \\
\hline
\end{tabular}

ALT - Alanine aminotransferase, BMI - Body Mass Index, CLIA - Chemiluminescent method, CLSI - Clinical and Laboratory Standards Institute, ECLIA - Electro-Chemiluminescence Immunoassay, ELISA Enzyme-Linked Immunosorbent Assay, FG - Fasting Glucose, FIRI - Fasting Insulin Resistance Index, FSI - Fasting Serum Insulin, GOD/POD - Glucose oxidase (GOD) and Peroxidase (POD) method, HOMA-IR - Homeostatic Model Assessment of Insulin Resistance, IFCC - International Federation of Clinical Chemistry and Laboratory Medicine, IRMA - Immunoradiometric method, ISSI-2 - Insulin Secretion-Sensitivity

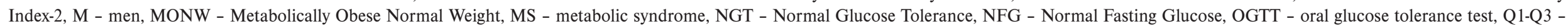
Interquartile Range, RI - Reference Interval, QUICKI -Quantitative Insulin Sensitivity Check Index, ROC - Receiver Operating Characteristic Curve, SD - standard deviation, W - women.

* the ratio of AUC insulin/glucose was calculated by traditional unites, $\mu \mathrm{IU} / \mathrm{mL}$ and $\mathrm{mg} / \mathrm{dL}$, respectively 
is defined mostly at 2.5 which results in an elevated frequency of insulin resistance, as we have seen also in our own research. Therefore, it is of paramount importance to determine a laboratory's reference intervals to properly identify insulin resistance ${ }^{41}$.

\section{The concept of reference range and methods of reference interval establishment}

The determination and introduction of common use reference intervals for IR indices is crucially important to doctors, general practitioners and other medical staff for better assessment of patients' clinical conditions and the application of appropriate therapeutic actions. The idea of reference intervals is based on the general concept of reference range, and the procedure used to carry it out is complex and multi-faceted ${ }^{43}$. The concept of reference range was launched by the Finnish researchers Ralph Gräsbeck and Nils-Erik Saris ${ }^{44,45}$ and based on comparison of the patients' results with a set of values used as reference, obtained on the basis of a well-described and standardized laboratory procedure. According to this concept, in order to establish reference ranges, it is necessary to provide: i) study population characteristics, the method of selecting subjects and assessing their health status; ii) criteria for the selection of subjects, their physiological condition, method of preparation for the study and procedure of collecting specimens; iii) the manner in which samples were handled and a description of the measurement, calculation and applied statistical methods ${ }^{46,47}$.

In 1969, the International Federation of Clinical Chemistry (IFCC) convened an Expert Panel on the Theory of Reference Values (EPTRV), in order to systemically develop the concept launched by Gräsbeck and Saris. EPTRV's tasks were to develop a procedure recommended for determining reference values, to analyse the obtained results and to establish appropriate nomenclature for their presentation ${ }^{48}$. The introduced terminology, intended for widespread use, is presented in Table 1. It includes the definitions and methods of determining reference intervals developed by the National Committee for Clinical Laboratory Standards (NCCLS), published in a document called C28-A3 in 2008 (ref. ${ }^{49}$ ).

The protocol presented in Table 1 refers to the a priori approach of selecting individuals for reference groups. This method consists in collecting data about a potential reference individual by means of a personal information questionnaire and health assessment, and the selection or exclusion of that subject. The last step, performed after all of the above procedures, is collecting the specimens. However, the second method of selecting reference individuals is the a posteriori approach, where the process of accepting the subject as a reference individual usually takes place after the collection of samples and laboratory examination. Both methods described above are referred to as direct sampling, which the IFCC recommends in the first instance for the determination of reference intervals. The a posteriori method could be also used for indirect methods of reference interval establishment based on previously collected data, known as data mining, which are especially useful for groups such as neonates and children $^{47,50}$.

\section{The reference intervals and cut off values for insulin resis- tance in different populations}

The majority of scientific studies provide information about insulin resistance indices values, given as mean or median, but only a few publications concern the determination of reference intervals according to the Clinical and Laboratory Standards Institute (CLSI) protocol and/or cut-off values for these indicators ${ }^{9,51,52}$. The most common values of insulin resistance indices used for the identification of these disturbances present in the scientific literature are established as upper 75 th or 95 th, or below 25 th or 5 th percentile values observed in the different examined populations. This is not exactly in accordance with laboratory guidelines, but is very widely applied in the scientific literature. In Table 2 the values of indices used to diagnose insulin resistance (HOMA1-IR, HOMA2, HOMA2 C-peptide, QUICKI), insulin sensitivity (Matsuda Index) and beta cell condition (ISSI-2), based on different decision-making factors, are presented in time order. We tried to present the most recent and different population studies in Table 2, and our aim was to show the variation in observed values rather than cite the results of all available studies.

As we show in Table 2, determination of cut-off values was applied not only to identify people with IR in the general population, but more frequently to differentiate populations with an increased risk of metabolic disorders associated with insulin resistance. This is understandable because IR is one of the most important factors in civilization diseases, such as DM, MS and CVD, as well as kidney failure ${ }^{73-76}$. Hence, knowledge of laboratory limitations and population differences in IR indices determination is important for the proper use of IR indices in making adequate clinical decisions. According to Table 2, the values for indirect insulin resistance indicators are different for different ages, sexes, populations and ethnic groups. The observed differences in the values of insulin resistance indices between different populations are related to ethnic heterogeneity ${ }^{77,78}$. One of the reasons for this situation may be the difference in the amount and distribution of body fat, body mass and height affecting BMI among the studied groups (i.e. Asians and Caucasians). According to our research results (in press) for young Caucasian (aged 18-31) the reference intervals for indirect insulin resistance indices which we examined according to CLSI protocol were: $\leq 4.00, \leq 2.27, \leq 4.10, \geq 0.31$ for HOMA1IR, HOMA2, HOMA2 C-pep., and QUICKI respectively. For insulin sensitivity the value of Matsuda Index was established as $\geq 3.19$ and for beta cell pancreatic function ISSI-2, as $\geq 206$. The cut-off values for insulin resistance recognition were established as 75 th percentile: $>2.78$, $>1.72,>2.63,<0.33$ for HOMA1-IR, HOMA2, HOMA2 C-pep., QUICKI respectively and $<4.31$ and $<261$ as 25 th percentile for Matsuda and ISSI-2, respectively ${ }^{72}$. In the scientific literature, various values of IR indicators are observed, not only due to individual or population vari- 
ability, but also the laboratory methods used to determine glucose and insulin concentrations.

The usefulness of HOMA indices are discussed in the literature and even the authors themselves indicate their limitations. Among others authors, Song et al. state that especially HOMA-IR, is independently and consistently associated with diabetes risk in a multiethnic cohort of U.S. postmenopausal women and using them could provide a benefits from early intervention in diabetes' high risk groups identified on the basis of HOMA indices ${ }^{79}$. At the same time Sung et al. criticized use HOMA-B\% for predicting diabetes in Korean women as useless, but they showed very good predictive values for HOMA1-IR and fasting glucose concentration to predicting diabetes ${ }^{80}$. Nearly twenty years after first describing in 2004, Wallace, Mathews et al. summaries utility of HOMA modeling in clinical and epidemiological studies. They maintained the opinion about usability HOMA derivate indices in predicting diabetes development, but also indicated ethnicity, reproducibility and reporting HOMA-B \% in isolation as the weak points of the model ${ }^{12}$.

On the basis of this study, it can also be observed that the presented reference intervals, decision values or cut-offs for insulin resistance indices are determined in various ways, but it is rare that they meet the criteria of the CLSI and/or IFCC recommendations. It should be emphasized that the CLSI document used to establish reference intervals, and even recommended for laboratory practice, is still not widely used in laboratory medicine.

However, currently the lack of an international standard for insulin laboratory determination, the use of different analytical methods, and the non-use of the CLSI protocol to receive reference intervals for indirect insulin resistance indicators all have a direct, limiting impact on the use of these parameters in an epidemiological context in routine medical practice. This is of particular importance to children and to adolescents, due to hormonal changes and the increase in and distribution of adipose tissue, parameters related to insulin resistance change.

Before introducing specific decision limits for the diagnosis of IR in clinical practice, appropriate reference values for indirect indices should be determined, and if this is not possible, data from studies carried out in the most ethnically, socio-economically and age-matched populations should be applied. The uncritical use of literature data without taking into account the values characteristic of the analyzed population may result in erroneous clinical decisions.

\section{CONCLUSION}

A comparison of insulin resistance and the sensitivity and efficiency of pancreatic $\beta$ cell values in different populations is difficult, due to significant differences in the results obtained by different analytical methods and the use of various criteria for inclusion and exclusion from the reference group. Hence, the decision-making factors for insulin resistance recognition should be applied judiciously, and the setting of certain recommended values for given populations and age groups is an urgent requirement.

In order to improve the diagnostic process for insulin resistance, population studies should be performed and the reference intervals and cut-off values for insulin resistance, insulin sensitivity and pancreatic $\beta$ cell function should be determined for different age groups, in accordance with CLSI protocol, and the standardization of laboratory methods of insulin measurement is indispensable. With regard to the enormous prevalence of diseases of civilization connected with insulin resistance, the issue of appropriate determination and knowledge of insulin resistance reference limit values by general practitioners is especially important for effective and accurate diagnosis and therapeutic measures.

\section{Search strategy and selection criteria}

Scientific articles from the period 1968 to 2018 were searched using the PubMed, SCOPUS and Google Scholar databases. Search terms included: insulin resistance, insulin resistance indicators, reference interval and decision limit. We wanted to assess the diversity in the range of indirect insulin resistance indices resulting from clinical trends, different laboratory methods and different populations and evaluated the proposed decision values and reference intervals.

Acknowledgement: This study was funded by a statutory grant awarded by the Ministry of Science and Higher Education, grant number: ST-D160.16.015.

Author contributions: SP, LPS, IK, AP: performed literature searches and participated in manuscript writing; AP, SP: made revisions and gave final approval of the manuscript; IK: was responsible for sending the final version. Conflict of interest statement: Authors declare that there are no conflicts of interest regarding the publication of this article.

\section{REFERENCES}

1. Abdul-Ghani MA, DeFronzo RA. Pathogenesis of Insulin Resistance in Skeletal Muscle J Biomed Biotechnol 2010;2010:476279. doi: 10.1155/2010/476279

2. Laakso M. Cardiovascular Disease in Type 2 Diabetes From Population to Man to Mechanisms: The Kelly West Award Lecture 2008. Diabetes Care 2010;33(2):442-9. doi: 10.2337/dc09-0749

3. Eckel RH, Kahn SE, Ferrannini E, Goldfine AB, Nathan DM, Schwartz MW, Smith RJ, Smith SR. Obesity and type 2 diabetes: what can be unified and what needs to be individualized? J Clin Endocrinol Metab 2011;96(6):1654-63. doi: 10.1210/jc.2011-0585

4. Friedrich N, Thuesen B, Jorgensen $T$, Juul A, Spielhagen C, Wallaschofksi $H$, Linneberg $A$. The association between IGF-I and insulin resistance: a general population study in Danish adults. Diabetes Care 2012;35(4):768-73. doi: 10.2337/dc11-1833

5. Ziaee A, Esmailzadehha N, Oveisi S, Ghorbani A, Ghanei L. The threshold value of homeostasis model assessment for insulin resistance in Qazvin Metabolic Diseases Study (QMDS): assessment of metabolic syndrome. J Res Health Sci 2015;15(2):94-100.

6. Qu HQ, Li Q, Rentfro AR, Fisher-Hoch SP, McCormick JB. The definition of insulin resistance using HOMA-IR for Americans of Mexican descent using machine learning. PLoS One. 2011;6(6):e21041. doi: 10.1371/journal.pone.0021041

7. Płaczkowska S, Pawlik-Sobecka L, Kokot I, Piwowar A. Estimation of 
metabolic factors related to insulin resistance and metabolic syndrome in young people. Scand J Clin Lab Invest 2018;78(4):325-32. https://doi.org/10.1080/00365513.2018.1469787

8. Gutch M, Kumar S, Razi SM, Gupta KK, Gupta A. Assessment of insulin sensitivity/resistance. Indian J Endocrinol Metab 2015;19(1):160-4 doi: 10.4103/2230-8210.146874

9. Tohidi M, Ghasemi A, Hadaegh F, Derakhshan A, Chary A, Azizi F. Ageand sex-specific reference values for fasting serum insulin levels and insulin resistance/sensitivity indices in healthy Iranian adults: Tehran Lipid and Glucose Study. Clin Biochem 2014;47(6):432-8. doi 10.1016/j.clinbiochem.2014.02.007

10. Bermudez V, Salazar J, Martínez MS, Chávez-Castillo M, Olivar LC Calvo MJ, Palmar J, Bautista J, Ramos E, Cabrera M, Pachano F, Rojas J. Prevalence and Associated Factors of Insulin Resistance in Adults from Maracaibo City, Venezuela. Adv Prev Med 2016;e9405105. doi: 10.1155/2016/9405105

11. Bermudez V, Rojas J, Martinez MS, Apruzzese V, Chavez-Castillo M, Gonzalez R, Torres Y, Salazar J, Bello L, Añez R, Chacín M, Toledo A, Cabrera M, Mengual E, Ávila R, Pachano F, López-Miranda J. Epidemiologic Behavior and Estimation of an Optimal Cut-Off Point for Homeostasis Model Assessment-2 Insulin Resistance: A Report from a Venezuelan Population. Int Sch Res Notices 2014;2014:616271. doi: 10.1155/2014/616271

12. Wallace TM, Levy JC, Matthews DR. Use and abuse of HOMA modeling. Diabetes Care 2004;27(6):1487-95. doi: 10.2337/diacare.27.6.1487

13. Hellgren MI, Jansson PA, Wedel H, Lindblad U. A lifestyle intervention in primary care prevents deterioration of insulin resistance in patients with impaired glucose tolerance: $A$ randomised controlled trial. Scand J Public Health 2016;44(7):718-25. doi: $10.1177 / 1403494816663539$

14. Appel SJ. Calculating insulin resistance in the primary care setting: why should we worry about insulin levels in euglycemic patients? J Am Acad Nurse Pract 2005;17(8):331-6. doi: 10.111/j.1745 7599.2005.0052.x

15. Muniyappa R, Lee S, Chen H, Quon MJ. Current approaches for assessing insulin sensitivity and resistance in vivo: advantages, limitations, and appropriate usage. Am J Physiol Endocrinol Metab 2008;294(1):15-26. doi: 10.1152/ajpendo.00645.2007

16. Pisprasert V, Ingram KH, Lopez-Davila MF, Munoz AJ, Garvey WT. Limitations in the use of indices using glucose and insulin levels to predict insulin sensitivity: impact of race and gender and superiority of the indices derived from oral glucose tolerance test in African Americans. Diabetes Care 2013;36(4):845-53. doi: 10.2337/dc12 0840

17. Santos JL, Yévenes I, Cataldo LR, Morales M, Galgani J, Arancibia C, Vega J, Olmos P, Flores M, Valderas JP, Pollak F.Development and assessment of the disposition index based on the oral glucose tolerance test in subjects with different glycaemic status. J Physiol Biochem 2016;72(2):121-31. doi: 10.1007/s13105-015-0458-0

18. American Diabetes Association. 2. Classification and Diagnosis of Diabetes: Standards of Medical Care in Diabetes-2018. Diabetes Care 2018;41(Suppl 1):S13-s27. doi: 10.2337/dc18-S002

19. Haneda $M$, Noda $M$, Origasa $H$, Noto $H$, Yabe D, Fujita $Y$, Goto A Kondo T, Araki E. Japanese Clinical Practice Guideline for Diabetes 2016. J Diabetes Investig 2018;9(3):657-97. doi: 10.1111/jdi.12810

20. 2017 Guidelines on the management of diabetic patients. A position of Diabetes Poland. Clin Diabet 2017;6SupplA:A1-A80. doi: 10.5603/ DK.2017.0001

21. Singh B, Saxena A. Surrogate markers of insulin resistance: A review. World J Diabetes 2010;1 (2):36-47. doi: 10.4239/wjd.v1.i2.36

22. Niemczyk S, Szamotulska K, Giers K, Jasik M, Bartoszewicz Z, Romejko-Ciepielewska K, Paklerska E, Gomółka M, MatuszkiewiczRowińska J.Homeostatic model assessment indices in evaluation of insulin resistance and secretion in hemodialysis patients. Med Sci Monit 2013;19:592-8. doi: 10.12659/MSM.883978

23. Matthews DR, Hosker JP, Rudenski AS, Naylor BA, Treacher DF, Turner RC. Homeostasis model assessment: insulin resistance and beta-cel function from fasting plasma glucose and insulin concentrations in man. Diabetologia 1985;28(7):412-9.

24. Levy JC, Matthews DR, Hermans MP. Correct homeostasis model assessment (HOMA) evaluation uses the computer program. Diabetes Care 1998;21(12):2191-2.

25. http://www.dtu.ox.ac.uk/homacalculator/ [Accessed 12.06.2018]
26. Katz A, Nambi SS, Mather K, Baron AD, Follmann DA, Sullivan G, Quon MJ.Quantitative insulin sensitivity check index: a simple, accurate method for assessing insulin sensitivity in humans. J Clin Endocrinol Metab 2000;85(7):2402-10. doi: 10.1210/jcem.85.7.6661

27. Kahn SE, Prigeon RL, McCulloch DK, Boyko EJ, Bergman RN, Schwartz MW, Neifing JL, Ward WK, Beard JC, Palmer JP,Quantification of the relationship between insulin sensitivity and beta-cell function in human subjects. Evidence for a hyperbolic function. Diabetes 1993;42(11):1663-72. doi: 10.2337/diab.42.11.1663

28. Matsuda M, DeFronzo RA. Insulin sensitivity indices obtained from oral glucose tolerance testing: comparison with the euglycemic insulin clamp. Diabetes Care 1999;22(9):1462-70.

29. Kahn SE, Suvag S, Wright LA, Utzschneider KM. Interactions between genetic background, insulin resistance and beta-cell function Diabetes Obes Metab 2012;14(Suppl3):46-56. doi: 10.1111/j.14631326.2012.01650.x

30. Bergman RN, Finegood DT, Ader M. Assessment of insulin sensitivity in vivo. Endocr Rev 1985;6(1):45-86. doi: 10.1210/edrv-6-1-45

31. Chen T, Xu F, Su JB, Wang XQ, Chen JF, Wu G, Jin Y, Wang XH. Glycemic variability in relation to oral disposition index in the subjects with different stages of glucose tolerance. Diabetol Metab Syndr 2013;5:38. doi: 10.1186/1758-5996-5-38

32. Blanco-Rojo R, Alcala-Diaz JF, Wopereis S, Perez-Martinez P, QuintanaNavarro GM, Marin C, Ordovas JM, van Ommen B, Perez-Jimenez F, Delgado-Lista J, Lopez-Miranda J. The insulin resistance phenotype (muscle or liver) interacts with the type of diet to determine changes in disposition index after 2 years of intervention: the CORDIOPREVDIAB randomised clinical trial. Diabetologia 2016;59(1):67-76. doi: 10.1007/s00125-015-3776-4

33. Retnakaran R, Shen S, Hanley AJ, Vuksan V, Hamilton JK, Zinman B. Hyperbolic relationship between insulin secretion and sensitivity on oral glucose tolerance test. Obesity (Silver Spring) 2008;16(8):1901-7. doi: 10.1038/oby.2008.307

34. Retnakaran R, Qi Y, Goran MI, Hamilton JK. Evaluation of proposed oral disposition index measures in relation to the actual disposition index. Diabet Med 2009;26(12):1198-203. doi: 10.1111/j.14645491.2009.02841.x

35. Allison DB, Paultre F, Maggio C, Mezzitis N, Pi-Sunyer FX. The Use of Areas Under Curves in Diabetes Research. Diabetes Care 1995; 18(2):245-50.

36. Henderson M, Rabasa-Lhoret R, Bastard JP, Chiasson JL, Baillargeon JP, Hanley JA, Lambert M. Measuring insulin sensitivity in youth: How do the different indices compare with the gold-standard method? Diabetes Metab 2011; 37(1):72-8. doi: 10.1016/j.diabet.2010.06.008

37. Borai A, Livingstone $C$, Kaddam I, Ferns G. Selection of the appropriate method for the assessment of insulin resistance. BMC Med Res Methodol 2011;11:158. doi: 10.1186/1471-2288-11-158

38. Ricos C, Perich C, Minchinela J, Alvarez V, Simon S, Biosca C, Doménech M, Fernández $P$, Jiménez CV, Garcia-Lario JV, Cava $F$. Application of biological variation - a review. Biochem Med (Zagreb) 2009;19:250-9.

39. Borai A, Livingstone C, Shafi S, Zarif H, Ferns G. Insulin sensitivity (Si) assessment in lean and overweight subjects using two different protocols and updated software. Scand J Clin Lab Invest 2010;70(2):98103. doi: $10.3109 / 00365510903544585$

40. Zozulińska D. Historia naturalna cukrzycy. Przew Lek 2006,3:30-9.

41. Płaczkowska S, Pawlik-Sobecka L, Kokot I, Piwowar A. Incidence of insulin resistance according to specified diagnostic criteria - a preliminary report. Hygeia Public Health 2014;49(4):851-6.

42. Płaczkowska S, Kokot I, Pawlik-Sobecka L, Piwowar A. Assessment of HOMA1-IR, Matsuda and ISSI-2 indices in relation to the metabolic syndrome features and oral glucose tolerance test in young people. Diagn Lab 2017;53(3):131-8.

43. Siest G, Henny J, Gräsbeck R, Wilding P, Petitclerc C, Queralto JM, Hyltoft Petersen $P$. The theory of reference values: an unfinished symphony. Clin Chem Lab Med 2013;51(1):47-64. doi: 10.1515/cclm2012-0682

44. Gräsbeck R, Saris NN. Establishment and use of normal values. Scand J Clin Lab Invest 1969;26:62-3.

45. Gräsbeck R, Fellman J. Normal values and statistics. Scand J Clin Lab Invest. 1968;21(3):193-5.

46. Gräsbeck R. Reference values, why and how. Scand J Clin Lab Invest Suppl 1990;201:45-53. doi: 10.1080/00365519009085800

47. Ozarda Y. Reference intervals: current status, recent developments 
and future considerations. Biochem Med (Zagreb) 2016;26(1):5-11. doi: 10.11613/BM.2016.001

48. International Federation of Clinical Chemistry, Scientific Committee, Clinical Section. Expert Panel on Theory of Reference Values (EPTRV). The theory of reference values. Part 5. Statistical treatment of collected reference values. Determination of reference limits. Clin Chim Acta 1984;137(1):97f-114f.

49. CLSI. Defining, Establishing, and Verifying Reference Intervals in the Clinical Laboratory; Approved Guideline-Third Edition. CLSI document C28-A3. Wayne, PA: Clinical and Laboratory Standards Institute; 2008

50. Appold K. Determining Laboratory Reference Intervals: CLSI Guideline Makes the Task Manageable. Laboratory Medicine 2009;40(2):75-6.

51. Yamada C, Mitsuhashi T, Hiratsuka N, Inabe F, Araida N, Takahashi E. Optimal reference interval for homeostasis model assessment of insulin resistance in a Japanese population. J Diabetes Investig 2011;2(5):373-6. doi: 10.1111/j.2040-1124.2011.00113.x

52. Takahara M, Katakami N, Kaneto H, Noguchi M, Shimomura I. Distribution of the Matsuda Index in Japanese healthy subjects. J Diabetes Investig 2013:4(4):369-71. doi: 10.1111/jdi.12056

53. Graffigna MN, Litwak L, Abdala MM, Akel ME, Aranda C, Gutt S, Ledesma L, Levalle O, Marcial Toro J, Migliano M, Pérez de la Puente M, Pombo F, Rodríguez M, Scaliter H, Tarruella M, Yuma María Cavallero E.Homa index in healthy subjects. Revista Argentina de Endocrinología y Metabolismo 2005;42(1):12-19.

54. Szurkowska M, Szafraniec K, Gilis-Januszewska A, Szybinski Z, Huszno B. Insulin resistance indices in population-based study and their predictive value in defining metabolic syndrome]. Przeg Epidemiol 2005;59(3):743-51.

55. Tresaco B, Bueno G, Pineda I, Moreno LA, Garagorri JM, Bueno M. Homeostatic model assessment (HOMA) index cut-off values to identify the metabolic syndrome in children. J Physiol Biochem 2005:61(2):381-8.

56. Keskin M, Kurtoglu S, Kendirci M, Atabek ME, Yazici C. Homeostasis model assessment is more reliable than the fasting glucose/insulin ratio and quantitative insulin sensitivity check index for assessing insulin resistance among obese children and adolescents. Pediatrics 2005;115(4):e500-3. doi: 10.1542/peds.2004-1921

57. Geloneze B, Vasques AC, Stabe CF, Pareja JC, Rosado LE, Queiroz EC, Tambascia MA; BRAMS Investigators. HOMA1-IR and HOMA2-IR indexes in identifying insulin resistance and metabolic syndrome: Brazilian Metabolic Syndrome Study (BRAMS). Arq Bras Endocrino Metabol 2009;53(2):281-7.

58. d'Annunzio G, Vanelli M, Pistorio A, Minuto N, Bergamino L, lafusco D, Lorini R, Arrigo T, Banin P, Cardella F, Cerutti F, Cherubini V, Chiarelli F, Cotellessa M, Crinò A, Meschi F, Toni S, Tonini G. Insulin resistance and secretion indexes in healthy Italian children and adolescents: a multicentre study. Acta Biomed 2009;80(1):21-8.

59. Gayoso-Diz P, Otero-Gonzalez A, Rodriguez-Alvarez MX, Gude $F_{\text {, }}$ Cadarso-Suarez C, Garcia F, De Francisco A.Insulin resistance index (HOMA-IR) levels in a general adult population: curves percentile by gender and age. The EPIRCE study. Diabetes Res Clin Pract 2011:94(1):146-55. doi: 10.1016/j.diabres.2011.07.015

60. Oka R, Yagi K, Sakurai M, Nakamura K, Moriuchi T, Miyamoto S, Nohara A, Kawashiri MA, Takeda Y, Yamagishi M. Insulin secretion and insulin sensitivity on the oral glucose tolerance test (OGTT) in middle-aged Japanese. Endocr J 2012;59(1):55-64. doi: 10.1507/ endocrj.EJ11-0157

61. Würtz P, Makinen VP, Soininen P, Kangas AJ, Tukiainen T, Kettunen J, Savolainen MJ, Tammelin T, Viikari JS, Rönnemaa T, Kähönen M, Lehtimäki T, Ripatti S, Raitakari OT, Järvelin MR, Ala-Korpela M.Metabolic signatures of insulin resistance in 7,098 young adults. Diabetes 2012;61(6):1372-80. doi: 10.2337/db11-1355

62. Yamada C, Moriyama K, Takahashi E. Optimal cut-off point for homeostasis model assessment of insulin resistance to discriminate metabolic syndrome in non-diabetic Japanese subjects. J Diabetes Investig 2012;3(4):384-7. doi: 10.1111/j.2040-1124.2012.00194.x

63. Stankiewicz-Olczyk J, Bolanowski M, Milewicz A, JawiarczykPrzybyłowska A. Częstość występowania zespołu metabolicznego u czynnych zawodowo mężczyzn. Endokrynologia, Otyłość Zaburzenia Przemiany Materii 2012;8(2):59-64.
64. Kozakowski J, Zgliczynski W. Body composition, glucose metabolism markers and serum androgens - association in women with polycystic ovary syndrome. Endokrynol Pol 2013;64(2):94-100.

65. Gayoso-Diz P, Otero-Gonzalez A, Rodriguez-Alvarez MX, Gude F, Garcia F, De Francisco A, Quintela AG.Insulin resistance (HOMA-IR) cut-off values and the metabolic syndrome in a general adult population: effect of gender and age: EPIRCE cross-sectional study. BMC Endocr Disord 2013;13:47. doi: 10.1186/1472-6823-13-47

66. Timoteo AT, Miranda F, Carmo MM, Ferreira RC. Optimal cut-off value for homeostasis model assessment (HOMA) index of insulin-resistance in a population of patients admitted electively in a Portuguese cardiology ward. Acta Med Port 2014;27(4):473-9.

67. Skoczeń M, Radwański K, Chrzanowska J, Zubkiewicz-Kucharska A, Seifert M, Noczyńska A. Carbohydrate and lipid metabolism disorders in children with simple obesity according to age and degree of obesity. Pediatric Endocrinology 2014;13(4(49)):27-36. doi: 10.18544/EP-01.13.04.1500

68. Bednarek-Tupikowska G, Zdrojowy-Welna A, Stachowska B, Kuliczkowska-Płaksej J, Matczak-Giemza M, Kubicka E, TworowskaBardzińska U, Milewicz A, Bolanowski M.Accumulation of abdominal fat in relation to selected proinflammatory cytokines concentrations in non-obese Wroclaw inhabitants. Endokrynol Pol 2014;65(6):44955. doi: 10.5603/EP.2014.0062

69. Oh TJ, Min SH, Ahn CH, Kim EK, Kwak SH, Jung HS, Park KS, Cho YM.Normal Glucose Tolerance with a High 1-Hour Postload Plasma Glucose Level Exhibits Decreased $\beta$-Cell Function Similar to Impaired Glucose Tolerance. Diabetes Metab J 2015;39(2):147-53. doi: 10.4093/dmj.2015.39.2.147

70. González-Zavala MA, Velasco-Morales A, Terrazas-Flores JJ, de la Cruz-Galicia MG, Cepeda-Nieto AC, Hernández-del Río A. Levels of insulin and HOMA-IR in adolescents in Saltillo, Coahuila, Mexico. Medicina Universitaria 2015;17(67):80-7. doi: 10.1016/j. rmu.2015.02.004

71. Kwon SS, Lee SG, Lee YH, Lim JB, Kim JH. Homeostasis model assessment of insulin resistance in a general adult population in Korea: additive association of sarcopenia and obesity with insulin resistance. Clin Endocrinol (Oxf) 2017;86(1):44-51. doi: 10.1111/cen.13233

72. Płaczkowska S, Pawlik-Sobecka L, Kokot I, Piwowar A. Estimation of reference intervals of insulin resistance (HOMA), insulin sensitivity (Matsuda) and insulin secretion sensitivity indices (ISSI-2) in Polish young people. Ann Agric Environ Med 2019; XX:XX-XX (in press).

73. Ormazabal V, Nair S, Elfeky O, Aguayo C, Salomon C, Zuniga FA. Association between insulin resistance and the development of cardiovascular disease. Cardiovasc Diabetol 2018;17(1):122. doi: 10.1186/s12933-018-0762-4

74. Reaven GM. Banting lecture 1988. Role of insulin resistance in human disease. Diabetes 1988;37(12):1595-607 doi: 10.2337/ diab.37.12.1595

75. Reaven GM. The role of insulin resistance and hyperinsulinemia in coronary heart disease. Metabolism 1992;41(5 Suppl 1):16-9. doi: 10.1016/0026-0495(92)90088-R

76. de Boer IH, Mehrotra R. Insulin resistance in chronic kidney disease: a step closer to effective evaluation and treatment. Kidney Int 2014;86(2):243-5. doi: 10.1038/ki.2014.123

77. Liu T, Li C, Shen L, Shen Y, Mao W, Li S. Heterogeneity in effects of genetically determined adiposity on insulin resistance and type 2 diabetes: The atherosclerosis risk in communities study. J Diabetes Complications 2018;32(3):330-4. doi: 10.1016/j.jdiacomp.2017.12.012

78. Vikram NK, Misra A, Pandey RM, Lithra K, Wasir JS, Dhingra V. Heterogeneous phenotypes of insulin resistance and its implicationsfor defining metabolic syndrome in Asian Indian adolescents. Atherosclerosis 2006;186:193-9. doi: 10.1016/j.atherosclerosis.2005.07.015

79. Song Y, Manson JE, Tinker L, Howard BV, Kuller LH, Nathan L, Rifa $\mathrm{N}$, Liu S.Insulin sensitivity and insulin secretion determined by homeostasis model assessment and risk of diabetes in a multiethnic cohort of women: the Women's Health Initiative Observational Study. Diabetes Care 2007;30(7):1747-52. doi: 10.2337/dc07-0358

80. Sung KC, Reaven GM, Kim SH. Utility of homeostasis model assessment of beta-cell function in predicting diabetes in 12,924 healthy Koreans. Diabetes Care 2010;33(1):200-2. doi: 10.2337/dc09-1070 